\title{
A Survey of Commercially Available Chemical Agent Instrumentation for Use in the Field
}

\author{
J. Haas, A. Alcaraz, B. Andresen, C. Pruneda
}

\section{March 1, 2002}

U.S. Department of Energy

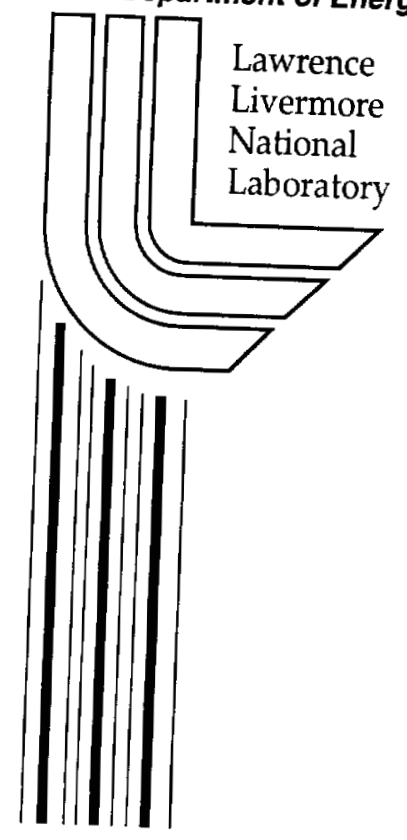




\section{DISCLAIMER}

This document was prepared as an account of work sponsored by an agency of the United States Government. Neither the United States Government nor the University of California nor any of their employees, makes any warranty, express or implied, or assumes any legal liability or responsibility for the accuracy, completeness, or usefulness of any information, apparatus, product, or process disclosed, or represents that its use would not infringe privately owned rights. Reference herein to any specific commercial product, process, or service by trade name, trademark, manufacturer, or otherwise, does not necessarily constitute or imply its endorsement, recommendation, or favoring by the United States Government or the University of California. The views and opinions of authors expressed herein do not necessarily state or reflect those of the United States Government or the University of California, and shall not be used for advertising or product endorsement purposes.

This work was performed under the auspices of the U. S. Department of Energy by the University of California, Lawrence Livermore National Laboratory under Contract No. W-7405-Eng-48.

This report has been reproduced directly from the best available copy.

Available electronically at http://www.doc.gov/bridge

Available for a processing fee to U.S. Department of Energy

And its contractors in paper from

U.S. Department of Energy

Office of Scientific and Technical Information

$$
\text { P.O. Box } 62
$$

Oak Ridge, TN 37831-0062

Telephone: (865) 576-8401

Facsimile: (865) 576-5728

E-mail: reports@adonis.osti.gov

Available for the sale to the public from

U.S. Department of Commerce

National Technical Information Service

5285 Port Royal Road

Springfield, VA 22161

Telephone: (800) 553-6847

Facsimile: (703) 605-6900

E-mail: orders@ntis.fedworld.gov

Online ordering: http://www.ntis.gov/ordering.htm

\section{OR}

Lawrence Livermore National Laboratory

Technical Information Department's Digital Library

http://www.llnl.gov/tid/Library.html 


\section{A Survey of Commercially Available Chemical Agent Instrumentation for Use in the Field}

Jeff Haas, Armando Alcaraz, Brian Andresen, and César Pruneda

Lawrence Livermore National Laboratory

7000 East Ave

Livermore, CA 94550

March-2002

Revision-1 


\section{Table of Contents}

Introduction 3

Project requirements 4

Survey of commercial CW Detectors

Color tests 4

Ion Mobility Spectroscopy (IMS)

Infrared Detection $\quad 8$

Surface Acoustic Wave Detection $\quad 10$

Flame Photometric 10

Mass spectrometers $\quad 12$

Sample collection and pre-concentration $\quad 12$

$\begin{array}{ll}\text { Preliminary Summary } & 13\end{array}$ 


\section{Principal Investigators:}

Jeffrey S. Haas

Phone: 925 422-6323

Fax: 925 423-5029

Email: haas1@llnl.gov

Brian D. Andresen

Phone: 925 422-0903

Fax: $925423-0914$

Email: andresen1@1lnl.gov
Armando Alcaraz

Phone: 925 423-6889

Fax: 925 423-9014

Email: alcaraz1@llnl.gov
César O. Pruneda

Phone: $422-0460$

Fax: 925 423-0914

Email:prunedal@llnl.gov

\section{Address:}

Forensic Science Center L-178

Lawrence Livermore National Laboratory

7000 East Ave

Livermore, CA 94550

\section{Introduction}

Lawrence Livermore National Laboratory's (LLNL) Forensic Science Center (FSC) has extensive experience and capabilities in the analysis of chemical agents (CA) and related compounds as well as experience in identifying these materials in the field (i.e. samples such as those found in soils, liquids, gases).

An open source survey was performed to determine viable, commercially available technology that can detect, in situ, CA and also meet field-use performance criteria as specified by the Program Management Consultant (PMC). The performance requirements of the technology include accuracy, reliability, integration onto robotics, and chemical detection sensitivities that meet required specifications.

Not included in this survey are technologies and methodologies to detect CA. decomposition products and related waste streams.

\section{Background of Researchers}

The principal investigators have in-depth knowledge and experience of CA environmental field analysis work. The FSC has developed instrumentation, methods, and procedures for the sampling, analysis, and detection of CA, explosives, drugs, nuclear materials, and characterization of many other chemical signatures. The FSC is also actively involved in the development of novel and rapid analysis techniques for many U.S. government entities and routinely deploys and implements the field analysis capabilities. 


\section{Project Requirements}

The intent of this project is to select or recommend CA monitoring system for the autonomous detection of CA in an excavation site. Table 1 lists the detection requirements for the autonomous detection system.

Table 1. Allowable Content Detection of Chemical Agent (with protective suit)

\begin{tabular}{|c|c|}
\hline Chemical Agent & Allowable Content $\left(\mathrm{mg} / \mathrm{m}^{3}\right)$ \\
\hline HD & 1 \\
\hline L & 0.06 \\
\hline DA/DC & 0.1 \\
\hline Chloroacetophenone $(\mathrm{CN})$ & 0.1 \\
\hline Phosgene & Under investigation \\
\hline Hydrogen Cyanide &
\end{tabular}

\section{Survey and Overview of Detection, Identification, and Verification of Chemical Agents by Commercially Available Technologies}

This is intended to be a survey and overview of commercial instrumentation and technology necessary for the detection of CA. Detection is vital in obtaining information on quantity of CA present and will be an important factor for implementing safe operational procedures at field sites contaminated with CA. Different types of detection scenarios will require different instrumentation and methods. For example, determining if $\mathrm{CA}$ agents are present in gas form and whether concentrations are at dangerous levels or not, or determining whether the soil, equipment, or surrounding munitions is contaminated with CA agent, and if it is at a level too dangerous to handle.

In order to reach decisions, it is necessary to know the type of CA present in the operations area and whether the concentration exceeds threshold levels. The awareness of potential danger through direct observations should be confirmed by some verification technique. The survey results are broken up into "Color Tests" and "Instrumentation".

\section{Color Tests}

Chemical detection paper is a very sensitive technique for detecting $\mathrm{CA}$. It is one of the least sophisticated and thus least expensive methods of detection. It is used to detect liquids and aerosols and is a common means for defining a contaminated area. Chemical detection paper is composed of 2 dyes soluble in $\mathrm{CA}$ and a $\mathrm{pH}$ indicator integrated into cellulose fibers. When exposed to CA, it can change color according to the type of agent. If an aerosolized droplet encounters the paper, the diameter and density of the spot can be used to determine the droplet size of the agent and the degree of contamination. 
This method is not entirely reliable, particularly if a long period of time has passed between the contamination and detection occasions. Considerable quantities of CA may have been absorbed into the soil layer and still imply danger without giving any response on detection paper. An example would be the chemical agent mustard, which forms micelles in liquids and in soils.

Also, chemical detection paper lacks specificity and is prone to error because it reacts with contaminants such as brake fluid, antifreeze, and insect repellent, resulting in falsepositive readings. False readings are especially undesirable in civilian situations because they may lead to erroneous decisions. However, CA identification can be done to some extent by means of a combination of manual vapor detection using tickets, color tubes, and color detection paper. Information on an even higher reliability level typically requires analysis in a laboratory setting. Since more detailed information with higher reliability on CA is typically necessary, instrumentation is required.

\section{M8/M9 Chemical Detection Paper}

Detection paper is based on certain dyes being soluble in CA. Normally, two dyes and one $\mathrm{pH}$ indicator are used, which are mixed with cellulose fibers in a paper without special coloring (unbleached). When a drop of CA is adsorbed into the paper, it will dissolve one of the pigments. For example, mustard agent will dissolve a red dye and a nerve agent will dissolve a yellow dye. In addition, VX nerve agent will cause the indicator to turn blue then, together with the yellow, will become green/green-black. Therefore, detection paper can thus be used to distinguish between three different types of CA. A disadvantage with the papers is that pesticides and other chemicals can also dissolve the pigments. Consequently, they should not be located in places where exposure to these materials is possible.

M8 and M9 CA detection papers, commonly used by the military, are available commercially to HAZMAT response teams. M8 paper is packaged in 25 perforated sheets, 2.5 in X 4 in, and is blotted on liquids that arouse suspicion. It identifies $\mathrm{CA}$ by changing colors within 30 seconds of exposure: dark green for vesicants, yellow for nerve agents, and red for blister agents.

M9 paper has adhesive backing that allows it to be attached to clothing and equipment. M9 paper detects the same agents as M8 paper but does not change color to enable identification. M9 paper tends to react faster than M8 paper and can be attached to vehicles that are entering areas filled with vapor to determine contamination. Vehicles thus equipped are limited to a speed of $30 \mathrm{~km} / \mathrm{h}$.

On the basis of spot diameter and density on the detection paper, it is possible to obtain an opinion on the original size of the droplets and the degree of contamination. A droplet of $0.5 \mathrm{~mm}$ diameter gives a spot sized about $3 \mathrm{~mm}$ on the paper. A droplet $/ \mathrm{cm}^{2}$ of this kind corresponds to a ground contamination of about $0.5 \mathrm{~g} / \mathrm{m}^{2}$. The lower detection limit in favorable cases is $0.005 \mathrm{~g} / \mathrm{m}^{2}$. 


\section{M256A1 Chemical Agent Detection Kit}

The M256 CA detector kit was originally released in 1978 and modified in 1987 to the M256A1; it is currently available commercially. This portable kit detects nerve gas, mustard gas, and cyanide and is usually used to define areas of contamination. The M256A1 contains a package of M8 paper, detailed instructions, and a vapor sampler (12 enzymatic tickets that contain laboratory filter paper for detecting CA vapors). The vapor sampler employs wet chemistry technology, in which ampoules containing different substrates are crushed so that the liquids interact with strips of filter paper, chromatographic media, and glass fiber filter. These substrates then are exposed to the vapor under suspicion. The reaction causes a color change, alerting the user to the presence of a CA. The reactions typically take 15 minutes to occur.

The M256A1 can detect nerve gas concentrations of $0.005 \mathrm{mg} / \mathrm{m}^{3}$, hydrogen cyanide concentrations of $11 \mathrm{mg} / \mathrm{m}^{3}$, and mustard gas concentrations of $0.02 \mathrm{mg} / \mathrm{m}^{3}$. This is one of the military's most sensitive devices for detecting CA and detects all agents at levels below those that can kill or injure people. It is prone to false-positive results, similar to other enzymatic detection techniques, but has not been demonstrated to produce falsenegative results in real situations.

\section{Colorimetric Tubes}

The detection tube for mustard agent is a glass tube containing silica gel impregnated with a substrate (DB-3). Detection air is sucked through the tube using a special pump. The reaction between the mustard agent and the substrate is faster by heating the tube. $\mathrm{A}$ developer is then added, and the result can be read. If the silica gel in the tube turns blue, then the vapor in the sample contains mustard agent (e.g. $\mathrm{Cl}(\mathrm{CH} 2) 2 \mathrm{~S}(\mathrm{CH} 2) 2 \mathrm{Cl}$ : mustard

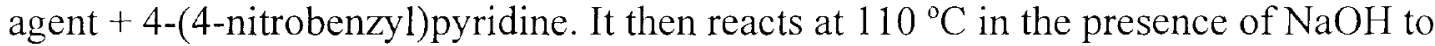
give 1-[1-[2-(2-chloroethylthio)ethyl]-1,4-dihydro-4-pyridylidenmethyl]-4-nitrobenzene, (blue).

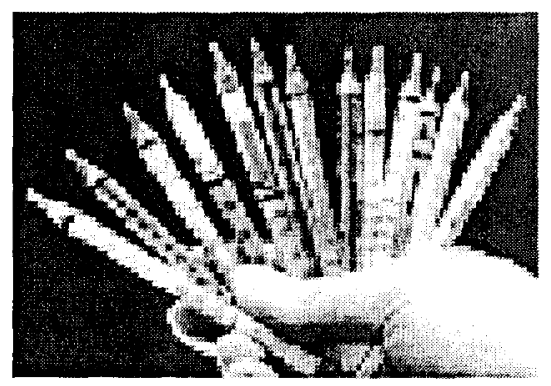

\section{Figure 1. MSA Gas Detection Tubes}

Colorimetric tubes such as those available from MAS Instrument Division, Draeger, and RAE systems use enzymatic techniques to identify CA. A hand pump is used to draw a sample into a specific tube, and the concentration of the substance is read from the tube. Available are 160 substance-specific reagent tubes identifying different agents. For each 
agent, a different tube must be used. Efficient use of this system demands knowledge of which CA is likely to be present in a given environment. If a tube for vesicants is used to sample the air and the CA is a nerve agent, the tube reports a false-negative result. A tube for each possible CA must be used for thorough detection.

Draegar has made this process simpler by offering a chip measurement system analyzer (CMS). The analyzer integrates an optical system for analyzing the color reaction, a flow controller, a pump system, and 10 capillaries, each capable of detecting an agent. As long as the proper chip is inserted, 10 agents can be detected and measured accurately within 20 seconds using this device. Draegar offers this device as part of an emergency response kit available to the public.

\section{Instrumentation}

This report focuses on detection technologies that are commercially available today. Many types of instrumentation are based on a number of different detection principles; these are described below.

\section{Ion Mobility Spectroscopy (IMS)}

IMS is used in many handheld and stand-alone detection devices that can be used to scan equipment, surfaces, and people for contamination. This technology involves drawing a gaseous sample into a reaction chamber using an air pump. The air molecules then are ionized, most commonly using radioactive beta emitters such as nickel-63 or americium241. The ionized particles then are passed through a weak electrical field toward an ion detector. Contaminants are identified according to the time it takes to traverse the distance to the detector. This time is proportional to the mass of the molecule. The pattern is compared to a sample of clean air; if the pattern is markedly different and unique to certain types of agents, the alarm sounds. These systems are capable of detecting and distinguishing between nerve gas, mustard gas, and vesicants. Its sensitivity ranges from $0.03 \mathrm{mg} / \mathrm{m}^{3}$ for nerve gases such as sarin to $0.1 \mathrm{mg} / \mathrm{m}^{3}$ for mustard gas.

IMS has certain advantages. It is less sensitive to contaminants, because it relies on a clean air sample for calibration. Thus, if an area has a certain baseline non-hazardous environmental vapor present, it is not detected.

The Finnish M86, M90, and the more recent ChemPro 100 are handheld devices that use IMS, as is the Improved Chemical Agent Monitor (ICAM). The ICAM was used extensively in the Gulf War, and even attached to certain vehicles. It is a handheld device that continuously displays the concentration of nerve agents or mustard agents. The ICAM is prone to erroneous detection in enclosed spaces and areas of strong vapor concentration (e.g., heavy smoke). It also can become saturated, requiring re-calibration. Versions of the ICAM are available for commercial purchase and are used by many medical response teams. 


\section{Figure 2. ICAM unit}

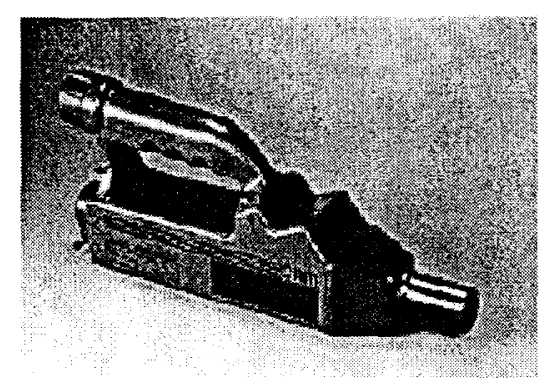

The APD 2000, manufactured by Environmental Technologies Group (ETG), is another common device that uses IMS and is sold commercially to HAZMAT response teams for domestic preparedness. This handheld device can be powered by batteries and can detect mace and pepper spray as well as nerve agents, blister agents, and hazardous compounds.

Many stand-alone detectors also use IMS technology. The military employs the M8A.1 detector that consists of a stand-alone detector, which continuously monitors the environment for hazardous vapors and aerosols, and up to 5 alarms that can be dispersed throughout an area. The M8A1 detects nerve agents and blister agents when the concentration is $0.1 \mathrm{mg} / \mathrm{m}^{3}$ or greater and sets off alarms within $1-2$ minutes. M8A1 is an ideal device for detecting a vapor released from an upwind direction. However, it is less effective for a vapor released in large amounts within seconds. In this situation, the alarm sounds after the personnel have been exposed. This system was used during the Gulf War and has been upgraded to the Automatic Chemical Agent Alarm (ACAA) system. The ACAA is slightly larger with a communications interface that is useful in combat.

ETG provides a commercial version of an IMS stand-alone detector called the Fixed Site/Remote Chemical Agent Detector. This system detects and identifies nerve and blister agents and offers superior reliability from interference. The alarm information can be transmitted via radio, satellite, or hardwiring.

\section{Infrared Detection}

This technique utilizes spectral methods, i.e., infrared (IR) for detection. Infrared radiation (IR) is employed in several CA detectors, including long-range detectors, (R\&D instrumentation in this area is currently being developed in the U.S. and France), and point detectors. IR can be used to excite molecules as each agent has a unique infrared pattern referred to as a fingerprint. Several different detection technologies utilize IR, including photoacoustic infrared spectroscopy, filter-based infrared spectroscopy, forward-looking infrared spectroscopy (FLIR), and Fourier transform spectroscopy.

\section{Photoacoustic Infrared Spectroscopy}

This highly selective technique is used to identify CA vapors. It usually is used in point detector devices. Modulated IR is passed through the sample. Since the CA absorbs the radiation, the temperature increases, and per Boyles law, the gas expands. The pattern of expansion or contraction depends on the modulation of the IR, which, in photoacoustic 
spectroscopy, is an audible signal. A microphone detects the modulation and alarms when it is similar to a recognizable agent.

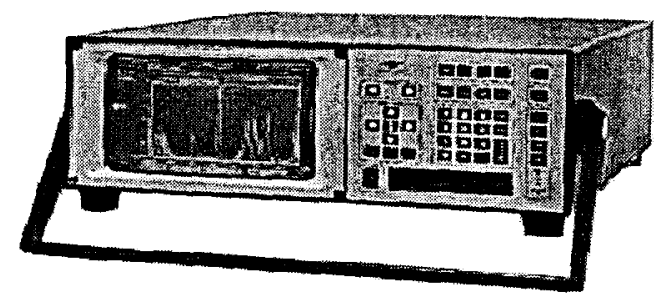

Figure 3. Innova 1301 Multigas Monitor

This technique's selectivity is based on the number of wavelengths transmitted through the sample. As more wavelengths are passed, the chance of contaminants causing false alarms decreases. These devices are sensitive to environmental variables such as external vibration. Like IMS, if these devices are calibrated in the operating environment, detection should be accurate.

\section{Filter-based Infrared Spectroscopy}

This technology also is based on comparing the amount of energy absorbed by the sample, using several different wavelengths of infrared light. A series of filters is used to direct the beam through a predetermined path. Concentrations of each vapor component are used to compile trends and identify the vapor.

\section{Differential Absorption Light Detection and Ranging}

This infrared technology is used mainly to track CA clouds that already have been identified. Two pulses of laser are transmitted into the distance, and the reflected IR is detected. One pulse is of a known frequency that is absorbed by the CA; the other is not known to be absorbed. The difference in the intensity of the return signal is used to determine the concentration of the cloud, while the time of return is used to determine the distance from the observers. This technique also is subject to environmental noise but has been used effectively to track CA.

\section{Passive Infrared Detection}

FLIR and Fourier transform infrared (FTIR) are techniques by which IR emitted from CA vapor is detected simply. These technologies commonly are used in stand-alone detection devices that simply alarm when a CA cloud is detected. Both of these methodologies depend on the collection of infrared information; however, the processing is different.

\section{M21 Remote Sensing Chemical Agent Alarm}

The military uses the M21 Remote Sensing Chemical Agent Alarm (RSCAAL) based on passive infrared detection. It is the first fielded standoff chemical detection device. This system can detect a vapor cloud from $5 \mathrm{~km}$ with an $87 \%$ detection rate. The M21 RSCAAL continuously monitors a background and notes the change in spectral information if a vapor cloud obstructs the background. It automatically scans along a $60^{\circ}$ angle, allowing the operator to monitor horizontal movement. The M21 can be set up in 
10 minutes and is unaffected by low light conditions. However, the M21 is limited in that it must be stationary and can be obstructed by snow and rain.

\section{Surface Acoustic Wave Detection}

Surface acoustic wave (SAW) chemical detectors rely on chemically selective coated piezoelectric crystals that absorb target gases. The absorption causes a change in the resonant frequency of the crystal that is measured by a microcomputer. These detectors are able to identify and measure many CAs simultaneously. These devices are produced inexpensively, making them a popular choice among civilian response units.

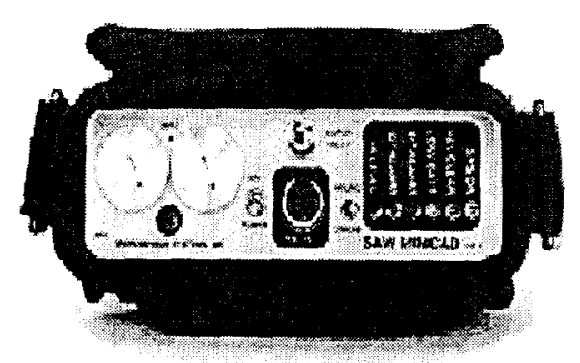

\section{Figure 4. The SAW MINICAD II}

The SAW MINICAD II is a portable SAW array detector that is lightweight, battery operated, and available commercially. It is used remotely to define areas of decontamination but also can be used for active detection.

\section{Flame Photometry}

Another technology employed is flame photometry. In this technique, a flame of hydrogen is used to burn a sample of air and detected by using a flame photometric detector (FPD). The color of the flame is analyzed by a photometer for concentrations of sulfur and phosphorous (key components in nerve gas and mustard). Flame photometry is highly sensitive yet is prone to false-positive results by detecting other gases that contain significant concentrations of sulfur or phosphorus but are non-hazardous. Certain analysis algorithms can be employed to make these detectors less prone to error.

If gas chromatography is integrated with flame photometry, the detectors are more accurate. Gas chromatography is a technique used in laboratories to separate mixtures of compounds. It involves using a carrier gas to separate a volatilized liquid or vapor based on its passage through a column. A detector records an electrical signal plotted over time as each solute exits the column according to its properties and the temperature of the column. Examples of instruments using this principle are the French monitor AP2C and Israeli combined detector and monitor CHASE.). 


\section{Miniature Automatic Continuous Agent Monitoring System}

The miniature automatic continuous agent monitoring system (MINICAMS) is a system based on combining gas chromatography with flame photometry. A sample vapor is drawn into the machine and exposed to a heated pre-concentrator loop. As each component exudes from the column, it is exposed to flame photometry. This system enables more specific detection. A typical cycle lasts 3-5 minutes, enabling continuous monitoring of the environment.

\section{Photo Ionization Detection}

Gas vapors can be ionized using ultraviolet light. Photo ionization detection depends on exposing the suspect vapor to ultraviolet energy powerful enough to ionize agent molecules. Specific ranges of ultraviolet light ionize molecules in certain CAs. An ion detector then registers the amount of ionized molecules. Thus, these detectors can determine the concentration and identity of the agent. The photoionization detector (PID) offers some advantages in ease of use, smaller size, and lower cost. In general, any compound with ionization energy lower than that of the PID lamp photons can be measured. Handheld detectors produced by RAE systems (MINIRAE Plus unit) and Photovac are examples of detectors that use this technology.

\section{Enzyme Detection Technology}

A research sector attracting great interest is the use of biologically active molecules as sensors. The approach is to use enzymes utilized in both manual modes of operation and in instrumental methods of detection. Detectors operating on this principle have been developed in the U.S.A., United Kingdom, Netherlands, and the former Soviet Union. The advantage of biosensors is that, at least in theory, they can be given the sensitivity and specificity desired. This is possible since the biosensor uses the same mechanisms that influence the human body when exposed to poisoning. A simple type of biosensor is the enzyme ticket.

Manual use of detection enzyme tickets for nerve agents are used in a similar way. The ticket consists of two parts, one with enzyme-impregnated paper and the other with substrate-impregnated paper. When the package is broken and the enzyme paper is wetted, the substrate part of the ticket is exposed to the test vapor by means of a pump.

Subsequently, the two parts are put together for two minutes. If the enzyme part of the ticket has turned a weak blue color, nerve agent is not present in the air. The detection limit is typically $0.02-0-05 \mathrm{mg} / \mathrm{m} 3$. The ticket can also be used without a pump (by waving it in the air) but this gives a slightly poorer sensitivity.

An example of the enzyme substrate reaction used in detection tickets for nerve agents can be seen below. Note that the blue change of color requires an active enzyme - some form of cholinesterase. In the presence of nerve agents, the enzyme is inhibited and no change of color occurs. Detection tickets of this kind cannot distinguish between the 
different nerve agents. For example, 2,6-dichloroindophenylacetate (red) + cholinesterase produces 2,6-dichloroindophenol (blue)

\section{Portable Gas Chromatograph-Mass Spectrometer (GC-MS)}

GC-MS analysis is routinely utilized in most analytical labs worldwide for the quantitative determination and the characterization of both known and total unknown chemicals. Most commercial GC-MS systems are non-portable laboratory-based systems, or are only transportable when the necessary support equipment (gas supply, vacuum system, computer, etc.)

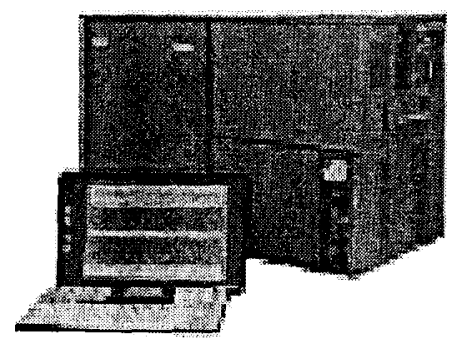

\section{Figure 5. Bruker Daltonics portable GC-MS (Viking 573)}

There are very few commercial portable GC-MS systems available but current user information has not been documented. The Bruker Daltonics portable GC-MS (Viking 573 ) is an example of a portable GC-MS system.

\section{Sample collection and pre-concentration}

The extraction of organic compounds from a sample matrix usually consists of purge-and-trap or headspace methods for concentrating volatiles; or liquid-liquid extraction, solid phase extraction (SPE), or supercritical fluid extraction for semivolatiles and non-volatiles. These typical methods of preparing samples for analysis by $\mathrm{GC}$ and GC-MS have various drawbacks, including requiring highly trained personnel, high costs, and excessive preparation time. A new sample preparation technique eliminates most drawbacks to sample preparation.

Solid phase microextraction (SPME) is one technology that can eliminates timeconsuming sample preparation in the laboratory or in the field. With this device, samples can be collected quickly by simply exposing SPME over the soil. SPME, therefore, requires no solvents or complicated apparatus. Almost immediately after SPME fiber exposure to the soil air, samples are ready for insertion into any GC or GC-MS injection port. One SPME fiber consists of a fused silica fiber coated with a thin film of solid phase adsorbent.

Several different solid phase adsorbents are currently available, and each can be obtained with different coating thickness. The type of sorbent material and coating thickness on the fiber is selective for a given class of compounds. Therefore, multiple fibers, each having a different sorbent/coating thickness, would cover a wide range of different 
classes of compounds encountered in field sampling scenarios (i.e., from volatile-low molecular weight to high-molecular weight polar compounds).

\section{Preliminary Summary}

A market survey of commercially available detection equipment in May 2000 identified 148 detection devices available to the military and first responders. With many different detectors and technologies available, the National Institute of Justice recommends examining 16 factors when choosing a detection device. Table 2 lists these factors. The most sensitive detectors tend to be most susceptible to false-positive alarms. Thus, for most practical applications, multiple detectors are needed to verify the findings of the initial detector.

In addition, a recent study by the U.S. National Guard recognized that no standards regulate the detection devices among different civilian emergency response units. Emergency teams can employ any of the devices and technologies described above. They commonly use inexpensive detectors such as SAW detectors and enzymatic techniques such as M9 paper and the M256 kit. Some teams also use IMS devices such as the APD2000 and a modified ICAM for domestic preparedness.

Table 2.

Factors to be Examined When Choosing a Detection Device

\begin{tabular}{|c|c|}
\hline Agents detected & Determination of agents most likely to be encountered \\
\hline Sensitivity & $\begin{array}{l}\text { Lowest concentration of CA that results in positive response; } \\
\text { ideally, lower than levels necessary for injury to personnel }\end{array}$ \\
\hline $\begin{array}{l}\text { Resistance to } \\
\text { interference }\end{array}$ & $\begin{array}{l}\text { Factors such as smoke, moisture, or other chemicals that prevent } \\
\text { the device from accurately providing a response }\end{array}$ \\
\hline Response time & Time to collect, analyze, and provide feedback \\
\hline Start-up time & Time to assemble and deploy the device \\
\hline Detection status & Vapor, liquid, and/or aerosols \\
\hline Alarm capability & Audible, visual, or both \\
\hline Portability & Ease of transport, which encompasses weight and dimensions \\
\hline Power capabilities & Battery versus alternating current \\
\hline Battery needs & Quantity and type of batteries \\
\hline $\begin{array}{l}\text { Operational } \\
\text { environment }\end{array}$ & Extremes of conditions under which the device operates \\
\hline Durability & Amount of abuse the device withstands \\
\hline Procurement costs & Cost per device needed \\
\hline Operator skill level & Skill involved in using the device \\
\hline $\begin{array}{l}\text { Training } \\
\text { requirements }\end{array}$ & $\begin{array}{l}\text { Number of hours and type of educational background required } \\
\text { for operation }\end{array}$ \\
\hline
\end{tabular}

This work was preformed under the auspices of the U.S. Department of Energy by Lawrence Livermore National Laboratory under contract W-7405-ENG-48. 